\title{
XX. Über isomorphe Mischsalze von wasserfreiem Kalk-, Magnesium- und Eisencarbonat.
}

\author{
Von
}

W. Diesel in Jena.

(Hierzu Taf. IV und 7 Textfguren.)

\section{Inhaltsverzeichnis.}

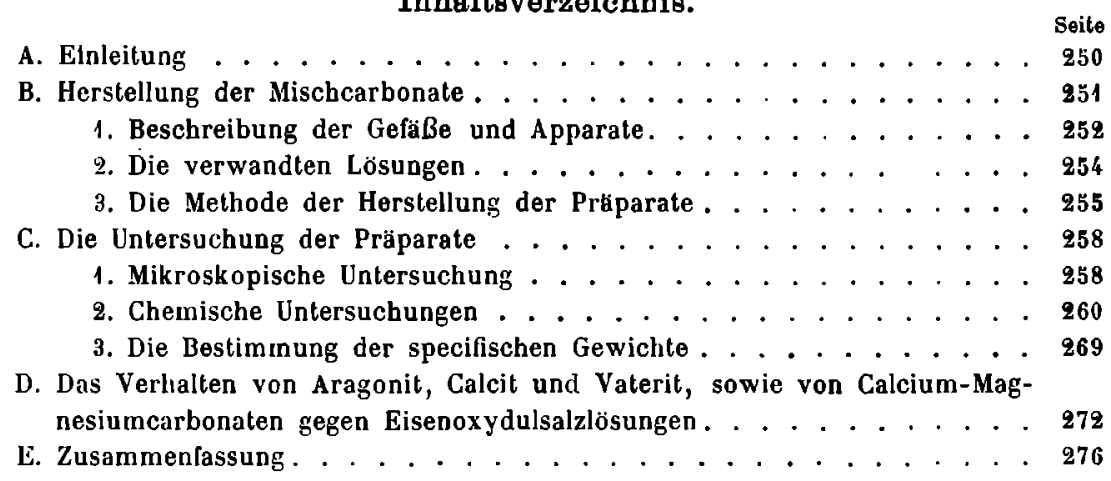

\section{Einleitung.}

Die Anregung zu den vorliegenden Untersuchungen erbielt ich durch Linck's Arbeit: „Über die Entstehung der Dolomite «, deren Resultate ich hier Kurz zusammenfassen möchte. Nach Linck ist der Dolomit das Product der Bildung eines chemischen Gleichgewichtes. Der fundamentale. Versuch, welcher zu diesem Resultate führte, war der folgende:

Es wurden ein Molekül Magnesiumchlorid

$$
\text { (d. h. } \frac{\mathrm{MgCl}_{2} \cdot 6 \mathrm{H}_{2} \mathrm{O}}{40}=\frac{203,26}{10} \mathrm{~g} \mathrm{MgCl}_{2} \cdot 6 \mathrm{H}_{2} \mathrm{O} \text { ) }
$$

und ein Molekül Magnesiumsulfat

$$
\text { (d. h. } \left.\frac{\mathrm{MgSO}_{4} .7 \mathrm{H}_{2} \mathrm{O}}{40}=\frac{146,42}{40} \mathrm{~g} \mathrm{MgSO}_{4} \cdot 7 \mathrm{H}_{2} \mathrm{O}\right)
$$


in $50 \mathrm{ccm}$ Wasser gelöst und dazu 1,5 Molekül Ammoniumsesquicarbonat

(d. h. $\left.\frac{\left(\mathrm{NH}_{4}\right)_{4} \mathrm{H}_{2}\left(\mathrm{CO}_{3}\right)_{3} \cdot \mathrm{H}_{2} \mathrm{O}}{40} \cdot \frac{3}{2}=\frac{272,16}{40} \cdot \frac{3}{2} \mathrm{~g}\left(\mathrm{NH}_{4}\right)_{4} \mathrm{H}_{2}\left(\mathrm{CO}_{3}\right)_{3} \cdot \mathrm{H}_{2} \mathrm{O}\right)$

in $150 \mathrm{ccm}$ Wasser gelöst gegeben. Diese klar bleibende Mischung wurde mit einer Lösung von 1 Mol. Calciumchlorid

$$
\text { (d. h. } \frac{\mathrm{CaCl}_{2}}{40}=\frac{111}{40} \mathrm{~g} \mathrm{CaCl}_{2} \text { ) }
$$

in $100 \mathrm{ccm}$ Wasser versetzt. Der entstandene gallertartige Niederschlag wurde unter Entweichen von Kohlensäure bei gewöhnlicher Temperatur langsam, bei höherer schneller krystallinisch und stellte in diesem Zustande ein aus Sphärolithen bestehendes, vollkommen einheitliches Mischcarponat aus ca. 1 Mol. Calciumcarbonat und 1 Mol.' Magnesiumcarbonat dar. Die Sphärolithe waren schwach positiv doppelbrechend, ihr specifisches Gewicht lag zwischen 2,6 und 2,7.

Ein vermehrter Zusatz an Calciumchlorid bedingte einen verminderten Magnesiumgehalt, dagegen wurde durch Vermehrung des Zusatzes an $\Lambda \mathrm{m}$ moniumsesquicarbonat der Gehalt an Magnesiumcarbonat erhöht: Die $\Lambda \mathbf{n}$ wesenheit von Natriumchlorid hatte eine Erniedrigung, die von Natriumcarbonat dagegen eine Erhöhung des Magnesiumgehaltes zur Folge.

Eine der Zusammensetzung des Dolomits fast genau entsprechende Substanz erhielt Linck dadurch, da $B$ er den auf oben angegebene Weise erhaltenen Niederschlag unter der Mutterlauge in einer zugeschmolzenen Röhre mehrere Stunden lang auf ca. $40^{\circ}-50^{\circ}$ erwärmte. Der Niederschlag bestand aus stark negativ doppelbrechenden Sphärolithen.

Linck hat also bei seinen Untersuchungen zuerst festgestellt, daß ein wasserfreies Mischsalz von Kalk- und Magnesiumcarbonat existiert.

\section{Herstellung der Mischcarbonate.}

Im Anschluß an die von Linck angestellten Untersuchungen über das Calcium-Magnesiumcarbonat wurde mir nun die Aufgabe gestellt, in dem aus Sphärolithen bestehenden Mischsalz das Magnesium in verschiedenem Maße durch Eisenoxydul zu ersetzen und die resultierenden dreifachen Mischcarbonate in chemischer und krystallographischer Hinsicht zu untersuchen.

Die Unbestăndigkeit der Eisenoxydulsalze bedingt eine verhältnismäßig complicierte Versuchsanordnung. Das Herstellen, Filtrieren und Trocknen des Niederschlags muB, um jede Oxydation zu verhindern” oder wenigstens zu verzögern, unter Luftabschluß vorgenommen werden. Als luftabschlieBendes und luftverdrängendes Mittel diente bei sämtlichen Versuchen Kohlensäure, welche einer Bombe entnommen wurde. Die so zur Verfügung 
stehende Kohlensäure wurde bei den ersten Versuchen direct benulzt. Der gewünschte Effect blieb jedoch aus. Es gelang nicht, einen nahezu unoxydierten Niederschlag zu erhalten. Die Ursache davon lag, wie sich bald herausstellte, in der Unreinheit der in der Bombe enthaltenen Kohlensäure. Sie enthielt einen ziemlich beträchtlichen Procentsatz Luft. Es galt also zunächst, die zur Verfügung stehende Kohlensäure von Luft zu befreien. Dies erreichte ich dadurch, daß ich sie über glühende Kupferdrahlnelzspiralen leitete. Die auf diese Weise gereinigte Kohlensäure wurde nun direct zu den einzelnen Versuchen benutzt.

\section{Beschroibung der Gefäße und Apparate.}

Bevor ich zur Schilderung der Versuche selbst übergehe, möchte ich eine kurze Beschreibung der benulzten Apparate und Gefäße vorausschicken.

Zur Herstellung und Aufbewahrung des

Fig. 1.

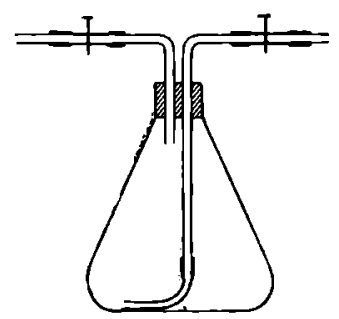
Niederschlags unter der Mutterlauge diente ein Erlenmeyer - Kolben (Fig. 1), welcher durch einen doppelt durchlochten Gummistopfen verschlossen ist. Durch diesen führen zwei Rühren in das Innere des Kolbens, von denen die längere den Boden des Gefäßes fast erreicht, während die kürzere dicht unterhalb des Stopfens mündet. Die längere Rübre trägt an dem inneren Ende einen Gummischlauch, welcher so lang ist, daß er bis zum Rande auf dem Boden des Gefäßes aufliegt. An die äußeren Enden der beiden Glasrühren sind ebenfalls kurze Gummischläuche angesetzt, die durch Quetschhähne verschlossen werden können.

Zum Filtrieren wurde ein Apparat benutzt,

Fig. 2.

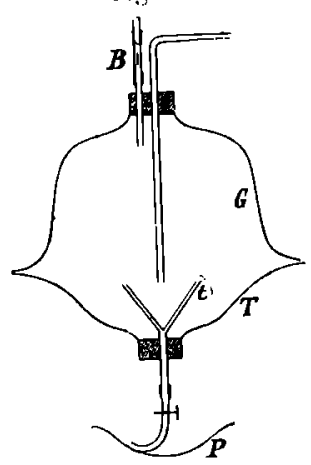
wie ihn Fig. 2 darstellt. $\mathrm{Er}$ bestebt aus einem Glasteller $(T)$, dessen Rand angeschliffen ist, und auf dem eine Glasglocke $(G)$ mit ebenfalls angeschliffenem Rande luftdicht aufsitzt. Der Glasteller hat in der Mitte eine Öfrnung, in welche ein einfach durchlochter Gummistopfen eingesetzt ist. Durch den Gummistopfen hindurch ragt in das Innere des Apparates ein Trichter $(t)$, welcher das Filter trägt. Die darüber gestülpte Glasglocke hat in ihrem obersten Teile eine Öffnung, welche einen doppelt durchbohrten Gummistopfen trägt. Durch diesen hindurch führen zwei Glasrühren. Die gebogene mündet dicht über dem Filter, die gerade dagegen schon im oberen Teile der Glocke und trägt an ihrem äußeren Ende ein Bunsen'sches 
Uber isom. Mischsalze von wasserfreiem Kalk-, Magnesium- u. Eisencarbonat. 253

Ventil $(B)$. An das Rohr des Trichters $t$ ist ein Gummischlauch angesetzt, welcher bis auf den Boden einer Porzellanschale $(P)$ reicht und durch einen Quetschhahn verschlossen werden kann. Nach einigen geringen Abänderungen dient der eben beschriebene Apparat zugleich zum Trocknen des Niederschlags.

Fig. 3.

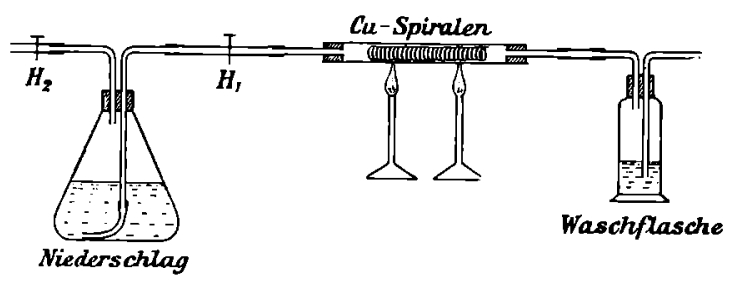

Zur Herstellung der Substanzen wurde die in Fig. 3 dargestellte Versuchsanordnung getroffen. Der Kohlensüurestrom gelangt aus der Röhre, welche die zur Reinigung der Kohlensïure dienenden Kupferdrahtnetzspiralen enthält, direct in den Erlenmeyer-Kolben (Fig. 1).

Zur Filtration des Niederschlags wurden die Apparate so angeordnet, wie Fig. 4 zeigt. Von der Glasröhre, welche die Kupferspiralen enthält,

liig. 4.

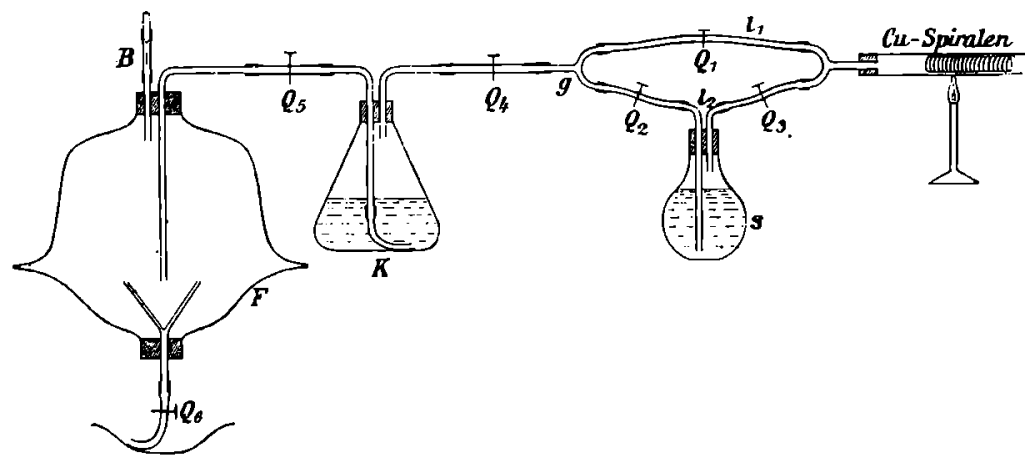

gehen zwei Kohlensäureleitungen aus, welche sich in $g$ wieder vereinigen. Die Leitung $l_{1}$ besteht aus einem Gummischlauch und kann durch den Quetschhabn $Q_{1}$ abgesperrt werden. In die Leitung $l_{2}$ ist eine gewöhnliche Spritzflasche $s$ eingeschaltet, welche durch die Quetschhähne $Q_{2}$ und $Q_{3}$ verschließbar ist. Die Spritzflasche $s$ enthält destilliertes Wasser, das vorher durch gründliches Auskochen von Luft befreit wurde. Von $g$ aus geht die Leitung weiter in den Erlenmeyer-Kolben $K$ (Fig. 1), welcher den krystallinischen Niederschlag enthält, und schließlich in den in Fig. 2 dargestellten Apparat 7 . 


\section{Die verwandten Lösungen.}

Die Concentrationen der bei den einzelnen Versuchen angewandten Lüsungen habe ich in Anlehnung an die von Linck gemachten Angaben gewählt. Nur müchte ich mich bei der Wiedergabe der Concentrationen einer etwas anderen Bezeichnung bedienen. Um eine kurze Darstellung der einzelnen Versuche zu ermöglichen, führe ich den in der Maßanalyse allgemein gebräuchlichen Bégriff der Normallüsung ein. Unter einer solchen Normallösung versteht man bekanntlich eine Lösung, welche im Liter das Äquivalentgewicht der betreffenden Substanz gelöst enthält. Eine normale Magnesiumchloridlösung würde demnach im Liter die Hälfte des Molekulargewichtes, also $101,63 \mathrm{~g} \mathrm{MgCl}_{2} \cdot 6 \mathrm{H}_{2} \mathrm{O}$, enthalten. Da ich bei meinen späteren Versuchen die Concentrationen stellenweise ändern muß, so werde ich in solchen Fällen hinter die Anzahl der Cubikcentimeter ein $(n),(2 n)$ (n/2) usw. setzen, um anzudeuten, daß die Lösung normal, doppeltnormal, halbnormal usw. ist. Es ist mir hierdurch die Möglichkeit gegeben, sowohl Versuchsbedingungen als auch Versuchsresultate bequem in Tabellen einzuordnen.

Die Lösungen, von welchen ich bei meinen Versuchen ausging, sind folgende:

I. $\frac{1}{2}$ normale Calciumchloridlösung. Sie enthält

$$
\frac{\mathrm{CaCl}_{2}}{4}=27,75 \mathrm{~g} \mathrm{CaCl} 2 \text { in } 1 \text { Liter. }
$$

IIa) normale Magnesiumsulfatlösung. Sie enthält

$$
\frac{\mathrm{MgSO}_{4} \cdot 7 \mathrm{H}_{2} \mathrm{O}}{2}=123,21 \mathrm{~g} \mathrm{MgSO}_{4} \cdot 7 \mathrm{H}_{2} \mathrm{O} \text { in } 1 \text { Liter. }
$$

b) normale Magnesiumchloridlösung. Sie enthält

$$
\frac{\mathrm{MgCl}_{2} \cdot 6 \mathrm{H}_{2} \mathrm{O}}{2}=101,63 \mathrm{~g} \mathrm{MgCl} \cdot 6 \mathrm{H}_{2} \mathrm{O} \text { in } 1 \text { Liter. }
$$

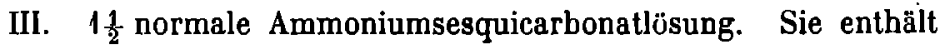
$\frac{\left(\mathrm{NH}_{4}\right)_{4} \mathrm{H}_{2}}{\left(\mathrm{CO}_{3}\right)_{9} \cdot \mathrm{H}_{2} \mathrm{O}} \cdot \frac{3}{6}=68,04 \mathrm{~g}$ Ammoniumsesquicarbonat in 1 Liter.

IV. Als Eisenoxydulsalz wurde das $\mathrm{Mohr}$ 'sche Salz $\mathrm{Fe}\left(\mathrm{NH}_{4}\right)_{2}\left(\mathrm{SO}_{4}\right)_{2} \cdot 6 \mathrm{H}_{2} \mathrm{O}$ verwandt. Da ich bei meinen Versuchen das Magnesium durch Eisenoxydul ersetzen will, so muß ich die Concentration der Mohr'schen Salzlüsung so wählen, daß sie so viel Ferrosulfat-Mole wie die Magnesialösung Magnesiumsulfat- bezw. Magnesiumchlorid-Mole enthält. Hieraus geht bervor, daß die Mohr'sche Salzlösung ebenfalls normal sein muB. Ein Liter Lüsung muß also $\frac{\mathrm{Fe}\left(\mathrm{NH}_{4}\right)_{2}\left(\mathrm{SO}_{4}\right)_{2} \cdot 6 \mathrm{H}_{2} \mathrm{O}}{2}=196,12 \mathrm{~g}$ Mohr'sches Salz
enthalten. 
Die Magnesiumsulfat- bezw. Magnesiumchloridlösung, welche Linck bei seinen Versuchen benutzte, war doppeltnormal, die von mir verwandte dagegen nur normal. Hätte ich die Magnesiumsalzlösung auch doppeltnormal gemacht, so hätte ich auch eine doppeltnormale Mohr'sche Salzlösung nötig gehabt, denn nur eine solche besitzt in bezug auf Ferrosulfat-Mole dieselbe Concentration wie eine doppeltnormale Magnesiumsalzlösung in bezug auf Magnesiumsulfat- bezw. Magnesiumchlorid-Mole. Ich hätte also eine Lösung herstellen müssen, welche im Liter 392,24 g Mohr'sches Salz enthält, eine Concentration, mit welcher sich wegen der Unbeständigkeit der Eisenoxydulsalze schwer arbeiten läßt. Die Concentrationen der Calciumchlorid- und der $\Lambda$ mmoniumsesquicarbonatlösung sind dieselben, wie sie Linck in seiner Arbeit angilst.

\section{Die Methode der Herstellung der Prïparate.}

Bei der Beschreibung der Herstellungsmethode der einzelnen Substanzen kann ich mich auf einen Versuch beschränken, da dieselbe Methode bei sämtlichen Versuchen wiederkehrt.

Der erste Versuch wurde auf folgende Weise ausgeführt:

Zunächst wurden $4.5 \mathrm{ccm}$ Magnesiumsulfat- bezw. Magnesiumchloridlösung (IIa bezw. IIb) mit "b ccm Mohr'scher Salzlösung (IV) in dem Erlenmeyer-Kolben (Fig. 1) vermischt. Ilierauf wurden $100 \mathrm{ccm}$ Calciumchloridlösung (I) und $150 \mathrm{ccm}$ Ammoniumsesquicarbonatlösung (III) gleichzeitig zu der Mischung von II und IV zugegossen und das Ganze tüchtig umgeschüttelt. Es entstand ein grünlich-grauer, dicker, gallertartiger Niederschlag, während aus der Flüssigkeit zahlreiche Kohlensäureblasen aufstiegen. Der Kolben wurde sofort nach der Fällung verschlossen. Hierauf wurde durch die bis auf den Boden des Kolbens führende Rühre unter fleiBigem Umschütteln von Luft vollständig befreite Kohlensäure eingeleitet. Die hierzu nötige Versuchsanordnung ist in Fig. 3 schematisch dargestellt.

War die Luft vollständig aus dem Erlenmeyer-Kolben verdrängt, so wurde der Kohlensäurestrom abgestellt, der Hahn $I_{1}$ geschlossen und bei $\mathrm{H}_{2}$ ein Bunsen'sches Ventil angesetzt. In diesem Zustande wurde das Gefäß3 ca. 15 Stunden stehen gelassen. Unter Entweichen von Kohlensäure wurde der Niederschlag krystallinisch. Nachdem die über dem Niederschlag stehende Flüssigkeit vollkommen klar geworden war und das Entweichen von Kohlensäure aufgehört hatte, wurde abfiltriert. Bei der Filtration wurde die in Fig. \& dargestellte Versuchsanordnung angewandt. Bevor die Filtration begann, wurde durch einen Verbindungsschlauch von $Q_{4}$ nach $Q_{5}$ in das Trichtergefäß $F$ Kohlensäure eingeleitet und aus demselben die Luft vollkommen verdrängt. Das Filtrieren selbst wurde eingeleitet durch Öffnen der Hähne $Q_{1}, Q_{4}$ und $Q_{5}$. Der in dem Erienmeyer-Kolben $\kappa$ 
befindliche Niederschlag wurde durch die Kohlensäure auf das Filter in $F$ hinübergedrückt. Mittels des Quetschhahnes $Q_{\theta}$ wurde der Abfluß des Filtrats so reguliert, daß das Filter immer zum größten Teile mit Flüssigkeit gefüllt war. Nach vollständiger Entleerung des Erlenmeyer-Kolbens

Fig. 5.

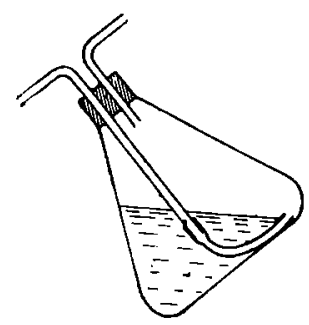
wurde der Hahn $Q_{1}$ geschlossen und $Q_{2}$ und $Q_{3}$ geöffnet. Zugleich wurde der Kolben $K$, wie Fig. 5 zeigt, auf die Kante gestellt, sodaß die Mündung des Gummischlauchs im Kolben möglichst hoch zu liegen kam. Durch die Kohlensäure wurde nun das im Kolben $s$ enthaltene, zum Auswaschen des Niederschlags bestimmte Wasser in den Erlenmeyer-Kolben gedrückt. Hatte das Wasser in $K$ die Schlauchmündung erreicht, so wurden die Hähne $Q_{2}$ und $Q_{3}$ wieder geschlossen und $Q_{1}$ geöfinet, zugleich wurde

der Kolben $K$ nach tüchtigem Umschütteln wieder in seine ursprüngliche Lage gebracht. Jas in $K$ angesammelte Wasser gelangte nun samt dem zurückgebliebenen Niederschlag infolge des Kohlensäuredruckes auf das Filter. Dieses Verfahren wurde so lange wiederholt, bis das bei $Q_{6}$ abfließende IFiltrat keine Salzsäure- bezw. Schwefelsäurereaction mehr zeigte. War dieser Punkt erreicht, so wurde an Stelle des Kolbens $s$ mit Wasser ein solcher mit absolutem Alkohol gesetzt, welcher ebenfalls vorher durch gründliches Auskochen von Luft befreit worden war. Der Niederschlag wurde nun zum Schluß nach dem angegebenen Verfahren noch einige Male mit Alkohol ausgewaschen. Es geschah dies, um das Trocknen des Niederschligs, das wegen der leichten Zersetzlichkeit desselben nicht durch Erwärmen bewerkstelligt werden konnte, zu erleichtern. Das Trocknen geschah durch Evacuieren. Das Filtriergefäß $F$ wurde zu diesem $Z$ wecke bei $Q_{5}$ und $Q_{6}$ abgeschlossen und nach Entfernung des Bunsen'schen Ventils bei $B$ mit einer Wasserstrahlluftpumpe verbunden. War das Trocknen soweit vorgeschritten; daß sich der Niederschlag von selbst vom Filter loslöste, so wurde die Substanz in ein Porzellanschälchen gebracht und in einem evacuierten Exsiccator so lange aufbewahrt, bis das Gewicht constant blieb. Substanzen mit geringerem Eisengehalt wurden nach dem Auswaschen mit Alkohol direct in den mit Kohlensäure gefüllten Exsiccator gebracht, worauf letzterer längere Zeit evacuiert wurde.

Auf diese Weise wurden die in den S. 262 usw. folgenden Tabellen I, II a, IIb, IIIa und IIIb angeführten Substanzen hergestellt. Jede dieser fünf Tabellen stellt eine Versuchsreihe dar. Die ersten vier Colonnen der Tabellen enthalten die bei der Făllung verwandten Mengen (in $\mathrm{ccm}$ ) der oben beschriebenen Ausgangslüsungen. In die nächsten drei Colonnen ist die Anzahl der zugesetzten Calcium-, Magnesium- und Eisen-Mole eingetragen. Die übrigen Spalten enthalten die Resultate der Analysen und die specifischen Gewichte. 
Betrachten wir zunächst die Versuchsreihe I, so sehen wir, daß die Menge des verwanden Ammoniumsesquicarbonats und Chlorcalciums bei sämtlichen sechs Versuchen beibehalten wurde. Sie beträgt 150 bezw. $100 \mathrm{ccm}$. Die Quantitälen des verwandten Magnesiumsalzes und Mohrschen Salzes dagegen wurden so verändert, daß die Summe der Cubikcentimeter stels 50 ergab. Es ist also bei sämtlichen Versuchen die Concentration an Calcium-, Magnesium- und Eisen-Molen constant erhalten worden.

Bei den Versuchen der Reihe IIa ist das Magnesium vollständig ausgeschaltet worden. Die Mengen an Calciumchlorid und Ammoniumsesquicarbonat wurden beibehalten und sind dieselben wie in der Tabelle $\mathbf{I}$. Dagegen wurde der Zusatz an Mohr'scher Salzlösung von $10 \mathrm{zu} 10 \mathrm{ccm}$ erhöht.

Im Anschluß an diese Versuchsreihe wurden noch die Versuche der Tabelle IIb ausgeführt. Auch hier wurde das Magnesium ganz ausgeschaltet. Der Unterschied zwischen dieser Versuchsreihe und der vorhergehenden besteht darin, daß hier die Concentration constant gehalten wurde, und zwar ist die Concentralion dieselbe, wie bei den in der Tabelle I angeführten Versuchen. Das Mohr'sche Salz veränderte ich wieder von 10 zu $10 \mathrm{ccm}$. Verwendete ich also im 12. Versuch $10 \mathrm{ccm}$ einer normalen Mohr'schen Salzlösung, so enlhalten diese $10 \mathrm{ccm} 10$ Mole Eisen. Die Summe der Magnesium-, Eisen und Calcium-Mole betrug bei den Versuchen der Tabelle I 100. Um nun diese Zahl beizubehalten, mußte ich als Ersatz für die $40 \mathrm{ccm}$ Magnesiumchloridlösung, welche 40 Magnesium-Mole enthalten, $40 \mathrm{ccm}$ einer normalen Calciumchloridlösung zusetzen. Auf diese Weise erreichte ich, daß bei den einzelnen Versuchen die Gesamtzahl der Mole und die Gesamtmenge der Flüssigkeit constant blieb. Führe ich dicse Betrachtung für alle fünf Versuche durch, so erhalte ich die Zahlen, welche in den ersten vier Colonnen angegeben sind. Die in runden Klammern beigefügte Bezcichnung bezieht sich auf die Concentration.

Zum Unterschied von den bisherigen Versuchsreihen wurde in der Tabelle IIIa das Calciumchlorid successive vermindert und zwar von $25 \mathrm{zu}$ $25 \mathrm{ccm}$, das Mengenverhältnis der verwandlen Magnesialösung zur Mohrschen Salzlösung aber constant erhalten.

In enger Beziehung zu der eben behandelten steht die Versuchsreihe IIlb. Auch hier wurde der Zusatz an Calciumchloridlösung gleichmäßig vermindert, hier jedoch außerdem dafür Sorge getragen, daß die Gesamtconcentration bei sämtlichen Versuchen constant blieb. Halten wir die beim Versuch 17 der Tabelle IIIa angewandte Concentration fest, so ändert sich an der Ausführung des Versuchs 22 nichts. Beim Versuch 23 verminderte ich den Zusatz an Calciumchlorid um $25 \mathrm{ccm}$ einer halbnormalen Lüsung, d. h. um 12,5 Calcium-Mole. Diese 12,5 Mole mußte ich 
an Magnesiumsalz- bezw. Mohr'scher Salzlösung wieder zusetzen, um die Concentration constant zu erhalten. Damit nun zugleich das Verhältnis zwischen den verwandten Mengen an Magnesiumchlorid und Mohr'schem Salz erhalten blieb, mußte ich 10 Magnesium-Mole, d. h. $20 \mathrm{ccm}$ einer halbnormalen Magnesiumchloridlösung, und 2,5 Eisen-Mole, d. h. $5 \mathrm{ccm}$ einer halbnormalen Mohr'schen Salzlösung zusetzen. Führen wir die entsprechende Betrachtung für die übrigen Versuche der Tabelle durch, so erhalten wir die Zahlen der ersten vier Colonnen.

\section{Die Untersuchung der Präparate.}

\section{Mikroskopische Untersuchung.}

Zwecks mikroskopischer Untersuchungen wurde nach dem Auswaschen mit Alkohol etwas von der im Kolben $K$ (Fig. 4) zurückgebliebenen Substanz in feuchtem Zustande mitlels eines Glasstabes auf einen Objectträger gebracht. Nachdem sämtlicher Alkohol verdunstet war, wurde die Substanz in eine Lösung von Canadabalsam in Chloroform eingebettet und mit einem Deckgläschen bedeckt.

Die mikroskopische Untersuchung ergab, daß es sich bei sämtlichen Versuchen um vollkommen einheitliche Substanzen handelte, die aus Sphärolithen bestanden (siehe Tafel IV). Diese Sphärolithe waren schwach doppelbrechend, zwischen gekreuzten Nicols zeigten sie farbige Ringe und ein schwarzes Kreuz. Der Charakter der Doppelbrechung erwies sich durchweg als negativ. Bei eisenreicheren Substanzen waren die Sphärolithe infolge der Oxydation schwach gelb gefärbt. Die gelbe Farbe war meist am Rande am intensivsten.

Ich lasse hier die Ergebnisse der mikroskopischen Untersuchung einiger Substanzen der Tabelle I folgen.

1. Versuch. Die Sphärolithe besitzen einen Durchmesser von ca. $13 \mu$. Fast alle zeigen einen mehr oder weniger trüben Kern und eine deutliche Zonarstructur. Die Anzahl der Zonen ist in den meisten Fällen 3. Bei kleiner Blendenöffnung ist fast bei allen Sphärolithen eine deulliche radialfaserige Structur zu bemerken. Letztere ist meist in der zweiten Zone am schärfsten ausgeprägt. Die Oberfläche der einzelnen Sphärolithe ist im allgemeinen rauh und zackig. Vollkommen kugelrunde Individuen sind selten. Sehr häufig kommen semmelförmige Verwachsungen vor.

2. Versuch. Der Durchmesser der Sphärolithe beträgt durchschnittlich $10 \mu$. Die äußere Begrenzung ist besser als bei den Substanzen des Versuches 1. Vollkommene Kugelformen sind häufiger. Die Zonarstructur ist ebenfalls sehr deutlich. Die Anzahl der Zonen beträgt 3 und mehr. Dagegen ist die radialfaserige Structur weniger deutlich. Fast jeder Sphärolith enthält einen trüben Kern. 
3. Versuch. Der Durchmesser der Sphärolithe ist ca. $9 \mu$ lang. Es herrscht hier eine auffallende Gleichmäßigkeit in der Größe der Sphärolithe. Die Doppelbrechung erscheint etwas stärker als bei (1) und (2). Alle Sphärolithe sind vollkommen rund und scharf begrenzt. Verwachsungen von mehreren Individuen sind viel seltener, auch das Auftreten eines trüben Kerns in den Sphärolithen ist bei weitem nicht mehr so häufig. Concentrisch-schalige und radialfaserige Structuren sind $\mathrm{zu}$ erkennen.

4. Versuch. Die Sphärolithe besitzen einen Durchmesser von 6 bis $8 \mu$. Die Begrenzung der einzelnen Individuen ist ausgezeichnet scharf. Trübe Kerne sind nicht vorhanden. Concentrisch-schalige und radialfaserige Structuren treten zurück. Meist ist überhaupt keine Structur zu erkennen, jedoch verrät das schwarze Kreuz' eincn radialfaserigen Bau. Solche Sphärolithe sehen aus wie kreisrunde, gleichmäßig gelb gefärbte Scheibchen. Zuweilen sind am Rande der Sphärolithe zwei ganz schmale Zonen zu erkennen.

Fassen wir die wichtigsten der an diesen vier Substanzen gemachten Beobachtungen zusammen, so ergibt sich:

Die Größe der Sphärolithe nimmt ab mit zunehmendem Eisengehalt. Die Doppelbrechung scheint bei eisenreicheren Sphärolithen stärker zu sein als bei eisenärmeren. Der Eisengehalt übt ferner auf die äußere Form der Sphärolithe einen deutlichen Einfluß aus. Eisenreichere sind regelmäBiger geformt und schärfer begrenzt als eisenarme. Concentrisch-schalige und radialfaserige Structuren werden bei zunehmendem Eisengehalt immer feiner und schließlich ganz unsichtbar. Ferner ist die Beobachtung gemacht worden, daß gerade solche Sphärolithe, welche einen Kern enthalten, die concentrisch-schalige und radialfaserige Structur am deullichsten zeigen.

Zu ganz ähnlichen Resultaten führte auch die Untersuchung der Substanzen der übrigen Tabellen. Ich will hier noch die Resultale der an den Substanzen der Tabelle IIa gemachten Untersuchungen kurz anführen:

7. Versuch. Die Größe der fast farblosen Sphärolithe ist sehr verschieden. Ihr Durchmesser schwankt zwischen 10 und $18 \mu$. Die äuBere Begrenzung der einzelnen Individuen ist teilweise sehr schlecht, die Formen sind unregelmäßig. Sehr zahlreich sind die Verwachsungen von kleineren und größeren Sphärolithen. Die concentrisch-schalige Structur ist besonders bei den größeren Sphärolithen sehr schön ausgebildet, auch sind trübe Kerne ziemlich häufig. Es sind meist drei Zonen vorhanden. Stellenweise ist auch die radialfaserige Structur sehr deutlich zu erkennen.

9. Versuch. Die Sphärolithe sind ziemlich groß. Sie erreichen einen Durchmesser von 20 ॥. Concentrisch-schalige und radialfaserige Structuren sind hier sehr gut zu sehen, dagegen treten die trüben Kerne in den Sphărolithen ziemlich selten auf. Die Ausbildung der Sphärolithe ist hier 
ausgezeichnet. Sie besitzen fast ausschließlich vollkommene Kugelgestalt. Merkwürdigerweise scheint jedoch hier die Zerselzung im Centrum der Sphärolithe begonnen zu haben.

11. Versuch. Der Durchmesser der Sphärolithe beträgt $\tau \mu$. Ganz vereinzelt sind auch etwas größere. Jeder Sphärolith ist kugelrund. Zonarstructur ist fast nicht zu sehen, nur stellenweise sind am Rande ein oder zwei ganz schmale Schalen zu erkennen. Die radialfaserige Structur ist so fein, daß sie überhaupt nicht zu erkennen ist. Hier scheint die gelbe Farbe am Rande der Sphärolithe intensiver zu sein.

Allgemein muß hier hervorgehoben werden, daß die Sphärolithbildung in den Tabellen II, wo das Magnesium fehlt, vollkommener ist als in den übrigen. Die Individuen sind im allgemeinen größer und zeigen die charakteristischen Structurarten deullicher.

Ich kann mich bei der Betrachtung der Resultate der mikroskopischen Untersuchungen auf diese beiden Tabellen beschränken, da die übrigen in dieser Hinsicht nichts neues bieten.

Zum Vergleiche habe ich auch einen reinen Ferrocarbonat-Niederschlag von der auf S. 268 angegebenen Zusammensetzung auf dieselbe Weise untersucht. Auch hier handelte es sich um oplisch negative Sphärolithe, die aber außerordentlich klein (Durchmesser $=4 u$ ), kugelrund und frei von Kernen sind. Von den beiden Structurarten ist nur die concentrischschalige zu erkennen, aber auch nur sehr selten. Das Vorhandensein der radialfaserigen Structur wird nur durch das schwarze Kreuz angezeigt.

An dieser Stelle möchte ich noch auf eine Erscheinung hinweisen, welche ich besonders an denjenigen Substanzen beobachtet habe, die mit Magnesiumsulfat hergestellt worden waren. Bei solchen Präparaten treten dic trüben Kerne in den Sphärolithen besonders häufig und groß auf. Oft hat es den Anschein, als ob der Kern aus winzigen Kryställchen bestände. Ich glaube deshalb die Annahıne machen zu dürfen, daß diese Kerne aus Gypskryställchen bestehen. Es haben sich wahrscheinlich bei der Fällung zunächst Gypskryställchen abgeschieden, um welche herum sich dann das Mischcarbonat abgesetzt hat. Ich werde bei der chemischen Untersuchung auf dieses Problem noch einmal zurückkommen.

\section{Chemische Untersuchungen.}

Zur Analyse wurden $0,3-0,4 \mathrm{~g}$ Substanz in überschüssiger verdünnter Salzsäure unter Erwärmen gelöst. Nachdem das Eisenoxydul durch Kochen mit einigen Tropfen rauchender Salpetersäure oxydiert worden war, wurde das Eisen mit Ammoniak als Hydroxyd gefällt und als Oxyd gewogen. Das Calcium wurde als Oxalat und das Magnesium als Pyrophosphat bestimmt. $\Lambda$ einer anderen Probe (ca. $0,3 \mathrm{~g}$ ) wurde aus dem Glühverlust der Gehalt an Kohlensäure und Wasser ermittelt. 
Als Beispiel führe ich eine ausführliche Analyse von der Substanz 2 der Tabelle I an. Die genannte Substanz wurde, nachdem sie ca. 24 Stunden (von der Filtration an gerechnet) im evacuierlen Exsiccator aufbewahrt worden war, jm Trockenschrank bei ca. $75^{0} 4$ Stunden lang getrocknet. Von dieser getrockneten Substanz wurden 0,3919 g zur Bestimmung der Basen und $0,3268 \mathrm{~g}$ zur Bestimmung des Glühverlustes verwandt. Zur Ermiltelung des Glühverlustes wurde die Substanz im offenen Tiegel bis zur Gewichtsconstanz geglüht. Das Resultat der Analyse war folgendes:

$$
\begin{aligned}
& 12,71 \% \mathrm{FeO} \\
& 40,64 \% \mathrm{CaO} \\
& 1,98 \% \mathrm{MgO} \\
& 44,38 \% \text { Glühverlust } \\
& \frac{99,71 \%}{4,78}
\end{aligned}
$$

Hierbei stellt die Zahl 44,38 bereits den corrigierten Glühverlust dar. Der durch Glühen unmittelbar festgeslellte Gewichtsverlust kann nicht direct zur Berechnung des Kohlensäure- und Wassergehaltes benutzt werden. Es muß berücksichtigt werden, daß beim Glühen das Eisenoxydul in Eisenoxyd übergeht $\left(2 \mathrm{FeO}+\mathrm{O}=\mathrm{Fe}_{2} \mathrm{O}_{3}\right)$. Es muß also von dem direct gefundenen Glühverlust die den $12,71 \%$ FeO äquivalente Menge Sauerstoff abgezogen werden. Auf diese Weise wurde die Zahl 44,38 erhalten. Dieser corrigierle Glühverlust gibt die Summe von Kohlensäure und Wasser an. Um nun den Wassergehalt selbst zu ermilteln, berechnete ich die Menge Kohlensäure, welche zur Bindung der gefundenen Oxyde nütig ist. Es ergeben sich folgende Werte:

$$
\begin{aligned}
& 12,71 \% \text { HeO brauchen } 7,77 \% \mathrm{CO}_{2} \\
& 40,64 \% \mathrm{CaO} \quad \times \quad 31,93{ }_{10} \mathrm{CO}_{2} \\
& \mathbf{1 , 9 8 \%} \mathrm{MgO} \quad, \quad \mathrm{z}, 16 \% \mathrm{CO}_{2} \\
& 41,86 \% \mathrm{CO}_{2}
\end{aligned}
$$

Durch Bildung der Differenz Glühverlust - Kohlensäure erhielt ich den Wassergehalt. Für unser Beispiel ergeben sich demnach

$$
\begin{aligned}
& 44,38 \% \text { Glühverlust } \\
&-41,86 \% \text { Kohlensäure } \\
& \hline \mathbf{2 , 5 2} \% \text { Wasser }
\end{aligned}
$$

Zur Prüfung der so erhaltenen liesultate wurden von einigen wenigen Substanzen, so auch von der Substanz 2 der Tabelle I, besondere Kohlensäure- und Wasserbestimmungen ausgeführt. $\mathrm{Zu}$ diesem Zwecke wurden ca. $0,5 \mathrm{~g}$ Subslanz in ein Porzellanschiffchen gebracht und in einer schwer schmelzbaren Glasröhre im Luftstrom geglüht. Das Wasser wurde durch festes Chlorcalcium und die Kohlensäure durch cine concentrierle Lösung von Kalilauge aufgefangen. Die Gewichtszunahme der Chlorcalciumröhren 
w. Diesel.

\begin{tabular}{|c|c|c|c|c|c|c|c|c|}
\hline & 怤 & & & $\begin{array}{l}\text { कू } \\
\text { on } \\
\text { on }\end{array}$ & & $\begin{array}{l}\frac{5}{5} \\
0 \\
\text { oi }\end{array}$ & & $\begin{array}{l}\overline{0} \\
\text { ò }\end{array}$ \\
\hline$\frac{0}{a}$ & $\begin{array}{l}\mathbf{\Xi} \\
.\end{array}$ & $\begin{array}{l}80 \\
0 \\
8\end{array}$ & $\underset{\substack{\infty \\
\hdashline}}{\infty}$ & 200 & 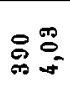 & 矢它 & $5:$ & 1 \\
\hline 옹 & 咅 & $\begin{array}{l}8 \\
8 \\
8 \\
5\end{array}$ & 용 & $\stackrel{\substack{0 \\
20 \\
\infty}}{\infty}$ & $\begin{array}{l}\overrightarrow{5}= \\
\overrightarrow{~ a d ~ a s ~}\end{array}$ & 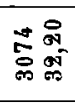 & 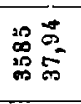 & 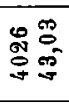 \\
\hline ह્ & T) & $8_{0}^{8}$ & 我 & 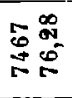 & क्ष & 경 $\frac{2}{20}$ & 象点 & 焉宫 \\
\hline & 疍 & $\begin{array}{l}8 \\
8 \\
8\end{array}$ & $\vec{D}$ & 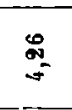 & 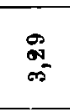 & $\sum_{\text {or }}$ & $\stackrel{20}{=}$ & 1 \\
\hline $\begin{array}{l}\vec{ت} \\
0 \\
0 \\
0\end{array}$ & 造 & $\overbrace{0}^{\infty}$ & 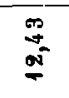 & $\frac{5}{5}$ & $\begin{array}{l}\text { N } \\
\infty \\
\infty \\
\text { ô }\end{array}$ & $\begin{array}{l}29 \\
20 \\
20\end{array}$ & $\frac{9}{25}$ & $\stackrel{8}{\circ}$ \\
\hline 总 & 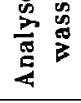 & $\begin{array}{l}\delta^{\infty} \\
0 \\
0\end{array}$ & $\begin{array}{l}\infty \\
\substack{\infty \\
20 \\
\text { of } \\
\infty}\end{array}$ & 5 & $\begin{array}{l}0 \\
5 \\
5 \\
0\end{array}$ & $\begin{array}{l}\text { बे } \\
\text { बे }\end{array}$ & 总 & 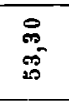 \\
\hline 总 & & 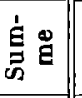 & $\frac{\infty}{0}$ & $\frac{5}{5}$ & $\begin{array}{l}\infty \\
\text { s. } \\
\text { g }\end{array}$ & $\begin{array}{l}\infty \\
: \\
8 \\
8\end{array}$ & $\begin{array}{l}\text { 怘 } \\
\text { 今 }\end{array}$ & $\vec{a}$ \\
\hline 邕总 & $\frac{0}{0}$ & 원 & के & 勇 & 5 & $\underset{\infty}{\infty}$ & $\begin{array}{l}30 \\
\infty^{n}\end{array}$ & 趈 \\
\hline 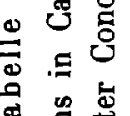 & $\begin{array}{l}\Xi \\
8 \\
0 \\
0 \\
0\end{array}$ & 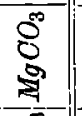 & $\vec{G}$ & $\equiv$ & $\underset{\infty}{\infty}$ & ơ & $\Sigma$ & 1 \\
\hline 鸹㽦 & $\stackrel{\square}{5}$ & $\overbrace{0}^{5}$ & $\frac{\infty}{\sim}$ & $\begin{array}{l}\infty \\
\stackrel{\infty}{0} \\
\infty\end{array}$ & 5 & 嗢 & $\begin{array}{l}8 \\
8 \\
0 \\
0\end{array}$ & $\begin{array}{l}8 \\
=\end{array}$ \\
\hline 范 & & 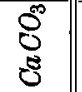 & $\begin{array}{l}\infty \\
\infty \\
\infty \\
\infty\end{array}$ & â & 5 & 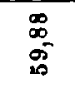 & $\overrightarrow{0}$ & 可 \\
\hline$\stackrel{\Phi}{ }$ & 党 & $\stackrel{8}{2}$ & $\stackrel{27}{7}$ & 9 & $\stackrel{20}{\circ}$ & 요 & 哭 & 오 \\
\hline 预 & 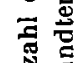 & $E^{\infty}$ & $\because 0$ & $\%$ & $: 2$ & ㅇ & ลื & 휴 \\
\hline & $\stackrel{3}{2}$ & 8 & : & มิ & 20 & 287 & : & in \\
\hline ه & 5 & 宽题 & 品 & $\stackrel{\circ}{\stackrel{8}{\circ}}$ & 足 & $\stackrel{20}{20}$ & in & ฆి \\
\hline 吉 & 虽 & $\begin{array}{c}\delta^{1} \\
0\end{array}$ & $\stackrel{\circ}{\div}$ & $\stackrel{9}{9}$ & $\stackrel{8}{\circ}$ & $\stackrel{8}{\circ}$ & $\stackrel{8}{\circ}$ & $\stackrel{8}{\circ}$ \\
\hline 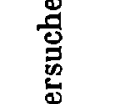 & 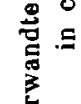 & 胥 & \& & $\stackrel{\circ}{\sim}$ & $\stackrel{2 n}{n}$ & 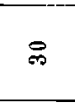 & ดั & 요 \\
\hline & 0 & 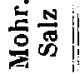 & $\therefore$ & $\underline{-}$ & $\stackrel{20}{=}$ & 워 & 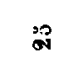 & 品 \\
\hline & & & - & ov & $\infty$ & $*$ & 20 & 0 \\
\hline
\end{tabular}


ergab den Wassergehalt und die des Kaliapparates den Kohlensāuregehalt. Die so gefundenen Resultate stimmten ziemlich gut mit den aus dem Glühverlust erhaltenen überein. Für das angeführte Beispiel wurde auf diese Weise ein Wassergehalt von 2,73\% festgestellt.

Die Colonnen 8-12 der Tabellen I-IIIb enthalten die directen Ergebnisse der Analysen in Procenten. In die Colonnen 13-15 sind die Procentzahlen der Analysen, bezogen auf wasserfreie Substanzen, eingeIragen. Für diese Zahlen sind die Molekularproportionen berechnet worden, welche in den nächsten drei Colonnen zusammengestellt sind.

Betrachtet man zunächst die Tabelle I, so erkennt man, daß das Ferrocarbonat außerordentlich leicht in das Calcium-Magnesiumcarbonat cintritt, und daß dies hauptsächlich auf Kosten des Magnesiumcarbonals geschieht. Aber auch der Gehalt an Calciumcarbonat wird bedeutend herabgedrückt. Der 6. Versuch der Tabelle liefert ein reines Calcium-Ferrocarbonat. Trotz eines Zusatzes von $20 \mathrm{ccm}$ Magnesialösung tritt hier kein Magnesium mehr in das Mischsalz ein.

Das Eisenoxydul wurde bei sämtlichen Versuchen vollständig gefällt, denn das Filtrat war bis auf geringe Spuren frei von Eisen. Dagegen blieb stets etwas Kalk und viel Magnesia in Lösung. Das geht auch aus den Versuchen der Tabelle I hervor, wenn wir dic Molekularproportionen mit der Anzahl der verwandten Calcium-, Eisen- und Magnesium-Mole vergleichen. $\mathrm{Zu}$ diesem $\mathrm{Zwecke}$ habe ich die Molekularproportionen (Quotient aus Procentzahl und Molekulargewicht) auf 100 berechnet und die so gefundenen Zahlen in den Tabellen unter die ejgentlichen Molekularproportionen gesetzt.

Für den Versuch 1 z. B. steht die Anzahl der verwandten Calcium-, Eisen- und Magnesium-Mole im Verhältnis 50:5:45=10:1:9. Im Niederschlag dagegen besteht zwischen der Anzahl der Calciumcarbonat-, Eisencarbonat- und Magnesiumcarbonat-Mole das Verhältnis 83:11:6. Vergleichen wir diese beiden Proportionen miteinander, so sehen wir, daß sowohl Kalk als auch Magnesia, von letzterer jedoch bedeutend mehr, in Lösung geblieben ist. Während in der Lösung vor der Fällung das Magnesium dem Eisen gegenüber in beträchtlichem Überschuß vorhanden war, überwiegt im Niederschlag das Eisen. Zu demselben Resultat gelangt man, wenn man diesen Vergleich für die übrigen Versuche dieser Tabelle durchführt. Auf den Wassergebalt der einzelnen Substanzen werde ich am Ende dieses Capitels etwas näher eingehen.

Bervor ich die chemische Betrachtung über diese Versuchsreihe schließe, muß ich noch auf eine gelegentlich gemachte Beobachtung hinweisen, welche mir bei meinen Untersuchungen zuweilen gewisse Schwierigkeiten machte. Im Capitel über die mikroskopischen Untersuchungen machle ich darauf 
aufmerksam, daß die trüben Kerne in den Sphärolithen teilweise beträchtlich groß sind und dann aussehen, als ob sie aus vielen kleinen Kryställchen beständen. Wie schon oben bemerkt wurde, handelt es sich ausschließlich um Substanzen, welche mit Magnesiumsulfat hergestellt worden waren. Solche Präparate lösten sich in kalter verdünnter Salzsäure nicht zu einer klaren Flüssigkeit, sondern ließen eine Trübung zurück, die beim Kochen vollständig verschwand. Versetzte man nun die klare salzsaure Lösung mit Chlorbaryum, so entstand eine Trübung. Es mußte also in den Mischsalzen Schwefelsäure enthalten sein. Es wurden auch einige Schwefelsäurebestimmungen ausgeführt, welche einen Gehalt bis zu 1\% ergaben. Alle Substanzen, welche diese Reaction zeigten, wurden zunächst von der weiteren Untersuchung ausgeschaltet und sind in der Tabelle nicht mit angeführt. Ich glaubte zunächst diese Unreinheit auf ein mangelhaftes Auswaschen zurückführen zu müssen, da andere, unter denselben Bedingungen angestellte Versuche vollständig schwefelsäurefreie Substanzen lieferten. Ich wiederholte deshalb das Auswaschen, die Schwefelsäurereaction war jedoch nicht zu beseitigen, ein Zeichen, daß die Erscheinung auf Einschlüsse der Sphärolithe an Mutterlauge oder Gyps zurückzuführen ist. Die Vermutung, daß die trüben Kerne aus Gypskryställchen bestehen, wird also hier gerechtfertigt. Die Substanzen, an welchen die eben behandelle Beobachtung gemacht wurde, waren, wie schon bemerkt wurde, ausschließlich mil Magnesiumsulfutlösung hergestellt worden. Zum Vergleiche wurden nebenbei auch einige Versuche unter Verwendung von Magnesiumchloridlüsung gemacht, welche in chemischer Minsicht dieselben Resultate lieferten. Die Verunreinigungen durch Schwefelsäure blichen jetzt bei den eisenarmen Substanzen ganz aus; bei den eisenreicheren waren sie $a b$ und zu noch zu bemerken, aber in weit geringerem Maße als bei den mit Magnesiumsulfat hergestellten Niederschlägen. Diese Beobachtung veranlaßte mich, bei meinen sämtlichen weileren Versuchen das Magnesiumsulfat auszuschalten und nur Magnesiumchlorid zu verwenden. In der Tabelle I sind ebenfalls nur mit Magnesiumchloridlüsung angestellte Versuche angeführt.

Von den Versuchen der Tabelle II a ist besonders Nr. 11 von Wichtigkeit. Bei diesem sind $50 \mathrm{ccm}$ Mohr'sches Salz und $100 \mathrm{ccm}$ Chlorcalcium verwandt worden. Da die Mohr'sche Salzlösung in bezug auf Ferrosulfat-Mole doppelt so concentriert ist wie die Chlorcalciumlösung in bezug auf Calciumchlorid-Mole, so sind gleich viel Ferrosulfat- und Calciumchlorid-Mole zugesetzt worden. Das resultierende Mischcarbonat besteht aus ca. $3 \frac{1}{2}$ Molekülen Calciumcarbonat und $5 \frac{1}{2}$ Molekülen Ferrocarbonat. Es ist aus dieser wie auch aus der Tabelle I zu ersehen, daß das Ferrocarbonat viel größere Neigung besitzt, mit Calciumcarbonat Mischcarbonale zu bilden als das Magnesiumcarbonat.

Die in der Tabelle IIb zusammengestellten Versuche Jiefern eine Reihe 
Über isom. Mischsalze von wasserfreiem Kalk-, Magnesium- u. Eisencarbonal. 265

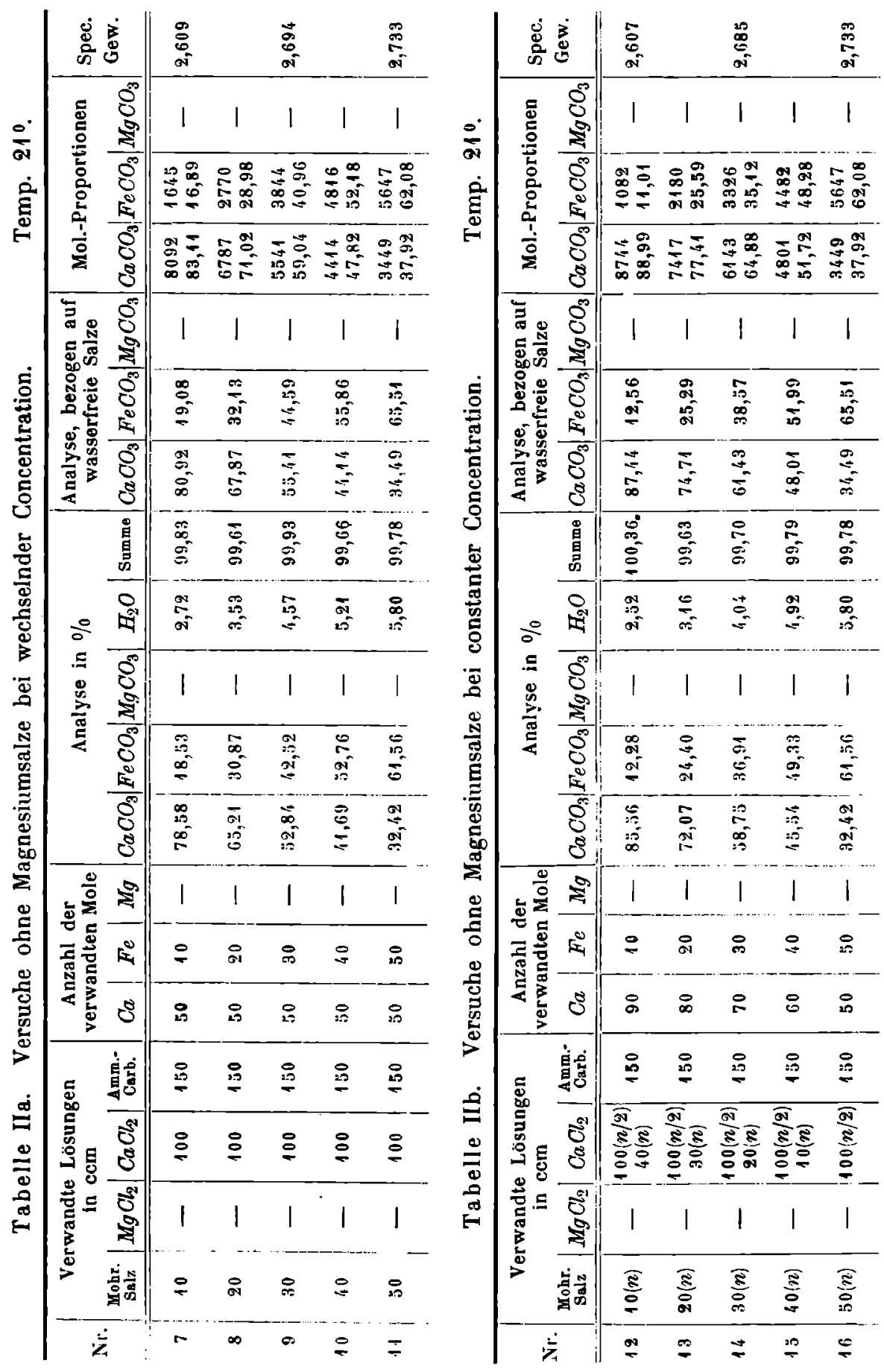


W. Diesel,

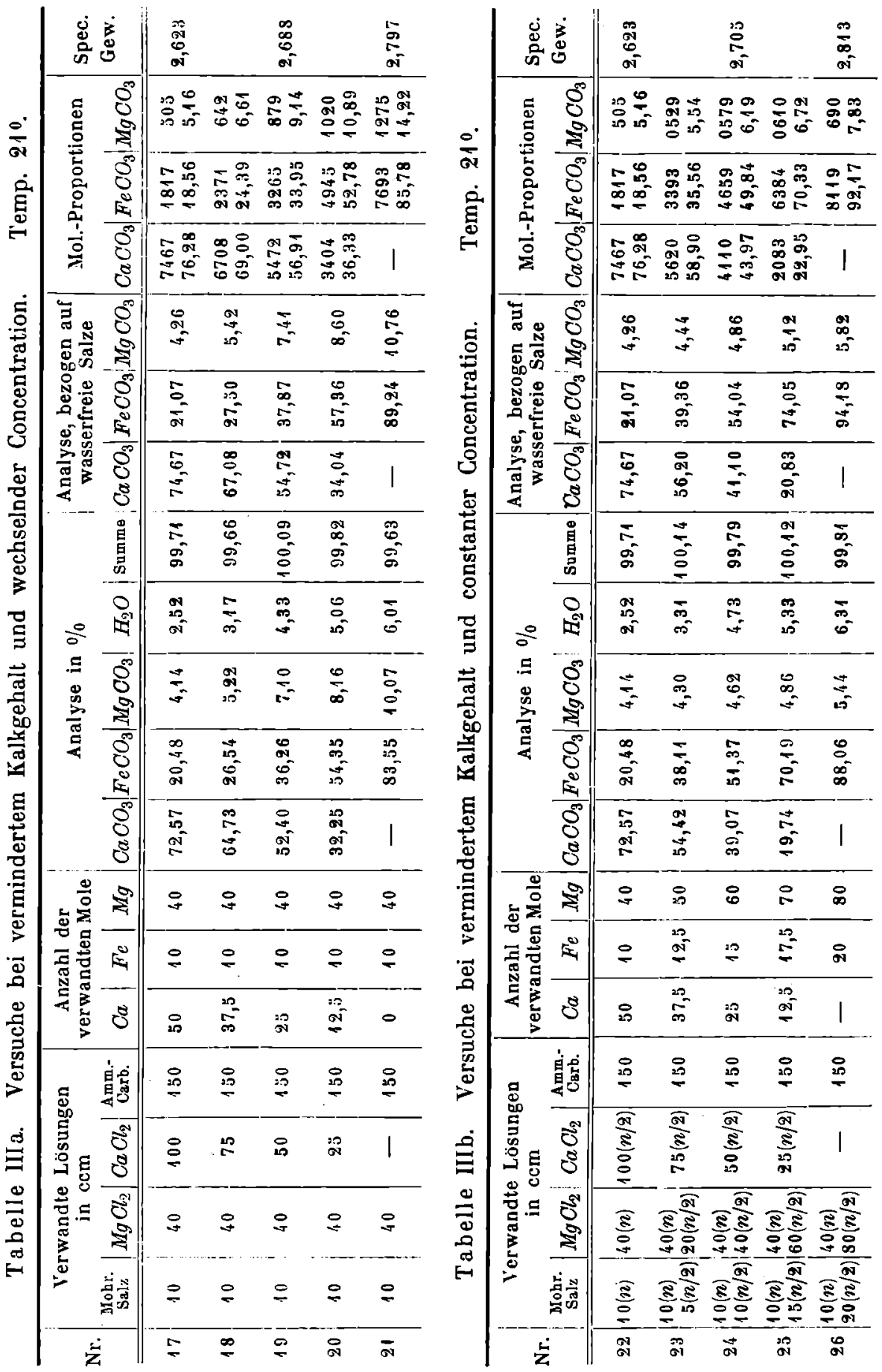


von Mischsalzen, in denen sich das Mengenverhältnis von Calciumcarbonat zu Ferrocarbonat stetig ändert. Der 16. Versuch der Tabelle ist identisch mit dem 11. Versuch in der Tabelle IIa.

Aus den Versuchsreihen IIa und IIb geht also hervor, daß sich Calciumcarbonat und Ferrocarbonat in allen Verhältnissen mischen.

Werfen wir einen Blick auf die Analysenresultate der Tabelle IIIa, so sehen wir, daß der Gehalt an Calciumcarbonat keineswegs gleichmäßig abnimmt, sondern daß die Abnahme immer stärker wird. Ferner beobachten wir, daß der Gehalt an Magnesiumcarbonat ziemlich gleichmäßig steigt, jedoch in verhältnismäßig geringem Maße. Es geht also aus diesen Versuchen hervor, daß das Calciumcarbonat zum weitaus grüßten Teile durch Ferrocarbonat ersetzt wird. Der 21. Versuch liefert ein FerroMagnesiumcarbonat aus ca. $\tau_{\frac{1}{2}}^{\frac{1}{2}}$ Molekülen Ferrocarbonat und 1 Molekül Magnesiumcarbonat.

Die Analysenresultate der Tabelle IIIb zeigen, daß der Gehalt an Calciumcarbonat zwar ständig abnimmt, aber durchaus nicht gleichmäßig. Der 22. Versuch ist identisch mit dem Versuch 2 der Tabelle I und 17 der Tabelle IIIa. Die geringste Differenz besteht zwischen (23) und (24). Sie beträgt $15,100_{i 0}^{\prime}$. Die Diflerenz zwischen (22) und (23) ist etwas größer und beträgt $18,47 \%$. Nahezu gleich sind die Differenzen zwischen (24) und (25) und zwischen (25) und (26). Sie betragen 20,27 bezw. 20,83\% . Entsprechende Unsteligkeiten finden wir in der Reihe des Eisencarbonats. Nahezu stetig ist dagegen die Zunahme des Magnesiagehaltes.

Bevor ich die chemische Betrachtung der Tabellen schließe, müchte ich noch etwas näher auf den Wassergehalt der einzelnen Substanzen eingehen.

Das Trocknen bewerkstelligte ich, wie schon oben bemerkt wurde, dadurch, daß ich die Substanz solange in einem evacuierten Exsiccator über concentrierter Schwefelsäure aufbewahrte, bis keine Gewichtsabnahme mehr festzustellen war. Einige in diesem Zustande analysierten Substanzen ergaben noch einen Wassergehalt von $6-70 / 0$. Ich suchte nun das weitere Trocknen durch Erwärmen im Trockenschrank herbeizuführen, was sehr oft eine Zersetzung der betreffenden Substanz zur Folge hatte. Auf diese Weise gelang es mir allerdings in den meisten Fällen, den Wassergehalt bedeutend herabzudrücken. Ferner versuchte ich mehrmals das Wasser dadurch zu entfernen, daß ich die Substanz einige Male mit Alkohol ausspülte und den Alkohol im Vacuum verdunsten ließ. Aber auch auf diese Weise kam ich nicht zum Ziele.

Aus sämtlichen Versuchen geht hervor, daß der Wassergehalt mit zunehmendem Eisengehalt zunimmt. Es müßte also zunächst angenommen werden, daß das Wasser als Kryslallwasser an das Eisencarbonat gebunden ist. 
Nach Dammer ${ }^{2}$ ) wurde von Sénarmont wasserfreies Eisenoxydulcarbontt als grauweißes, aus Rhomboëdern bestehendes Pulver durch 36 stündiges Erhitzen von Eisenvitriollösung mit Natriumbicarbonat auf $150^{\circ}$ und darüber, ferner aus Eisenchlorür und Calciumcarbonat bei $200^{\circ}$ gewonnen. Außer diesem wasserfreien Eisencarbonat ist noch ein wasserhaltiges von der Zusammensetzung $\mathrm{FeCO}_{3}+\mathrm{H}_{2} \mathrm{O}$ bekannt. Nach Dammer wird es durch Füllen einer Eisenoxydulsalzlösung mit Natriumcarbonal, Auswaschen und Trocknen bei Luftabschluß als grünweißes oder weißes Pulver erhalten. Durch Fällen von $100 \mathrm{ccm}$ einer doppeltnormalen $\mathrm{Mohr}$ schen Salzlüsung mit $150 \mathrm{ccm}$ einer $1 \frac{1}{2}$ normalen Ammoniumsesquicarbonatlüsung bei 220 erhielt ich ein Product von der Zusammensetzung:

$$
\begin{gathered}
89,91 \% \mathrm{FeCO} \\
9,62 \% \mathrm{H}_{2} \mathrm{O} \\
99,53 \%
\end{gathered}
$$

Diese Verbindung bestand aus mikroskopisch kleinen Sphärolithen. Das wasserhaltige Eisenoxydulcarbonat mil einem Molekül Wasser würde die Zusammensetzung:

$$
\begin{array}{lll}
86,56 \% & \mathrm{FeCO} & \\
13,44 \% & \mathrm{H}_{2} \mathrm{O} & \text { besilzen. }
\end{array}
$$

Fragen wir uns nun: welche von diesen beiden Verbindungen ist in den vorliegenden Mischsalzen enthalten? Bevor ich an die Beantwortung dieser Frage gehe, muß ich eine Beobachtung anführen, welche bei der Lösung dieses Problems berücksichtigt werden muß. Es zeigle sich nãmlich, daß der Wassergehalt durch Verminderung des Zusatzes an Eisensalz nie unter $2 \%$ heruntergedrückt werden kann. Ebenfalls aus Sphärolithen beslehende Calcium-Magnesiumcarbonate sind von Schmid( 3 ) auf ganz analoge Weise hergestellt worden. Dieselben ergaben einen Wassergehalt von ca. $2 \%$. Wenn wir annehmen, daß sämtliches Wasser an das Eisencarbonat gebunden ist, so reicht es doch nicht aus, um mit Ferrocarbonat zusammen die Verbindung $\mathrm{FeCO}_{3}+\mathrm{H}_{2} \mathrm{O}$ zu bilden. Zum Beweis führe ich die Molekularproportionen für Eisencarbonat und Wasser einiger Substanzen an.

$$
\mathrm{HeCO}: \mathrm{H}_{2} \mathrm{O}
$$

$$
\begin{array}{crl}
\text { Versuch } 2: & 1765: 1400=1: 0,8 \\
- & 5: & 3439: 2138=1: 0,6 \\
- & 9: & 3666: 2539=1: 0,7 \\
-\quad 14: & 3182: 2244=1: 0,7 \\
-\quad 20: & 4685: 2811=1: 0,6 \\
-\quad 24: & 4604: 2600=1: 0,56
\end{array}
$$

Die Existenz eines Eisencarbonats mit weniger als einem Molekül Wasser ist sehr unwahrscheinlich. Da nun, wie die mikroskopische Unter- 
suchung gezeigt hat, die radialfaserige Structur mit zunehmendem Eisengehalt immer feiner wird, so glaube ich die Annahme machen zu dürfen, daß das sämlliche Wasser von den Sphärolithen eingeschlossen wird. Die feine und dichte Structur bedingt, daß das Wasser beim Trocknen der Substanzen aus dem Innern der Sphärolithe nicht entweichen knnn.

\section{Die Bestimmung der specifischen Gewichte.}

Zur Bestimmung der specifischen Gewichte wurde die Vorrichtung gebraucht, welche Fig. 6 darstellt. Es handelt sich im-wesentlichen um zwei Glascylinder $C_{1}$ und $C_{2}$. Der $\mathrm{Cy}$ linder $C_{1}$ trägt in einer Höhe von $8 \mathrm{~cm}$ Fig. 6. vom Boden des Gefäßes aus gerechnet eine Zuflußröhre, die millels eines Glashahnes $H$ verschlossen werden kann. Der ganze Cylinder ist verschlossen durch einen doppelt durchbohrten Korkstopfen, in welchen zwei Glasröhrchen, eine gerade $R_{1}$ und eine gebogene $R_{2}$, eingesetzt sind. Um ein luftdichtes Schließen des Stopfens zu bewirken,

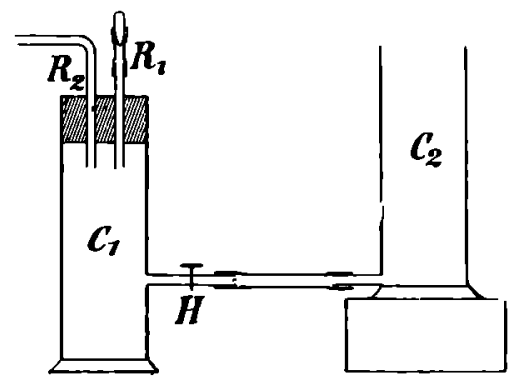
ist dieser noch mit einer Schicht Siegellack bedeckt. Der Cylinder $C_{2}$ ist offen und trägt dicht über dem Boden ebenfalls ein Ansatzrohr. Die Verbindung beider Cylinder wird durch einen Gummischlauch hergestellt. Die einzelnen Bestimmungen wurden auf folgende Weise ausgeführl:

Die Substanz wurde durch die Röhre $R_{1}$ in den sorgfältig getrockneten Cylinder $C_{1}$ gebracht und die Röhre $R_{1}$ durch einen Gummischlauch mit Glasslopfen luftdicht verschlossen. Die Röhre $R_{2}$ wurde mit der Luftpumpe verbunden und, nachdem der Glashahn $H$ geschlossen worden war, der Cylinder $C_{1}$ luftleer gepumpt. Dieses Verfahren halte den Zweck, die von der feinpulverigen Substanz festgehaltene Luft zu entfernen. Das Evacuieren wurde mehrmals wiederholt und unterdessen in den Cylinder $C_{2}$ eine Thoulet'sche Lösung gebracht, welche nahezu das specifische Gewicht der zu untersuchenden Substanz besaß. Durch Öffnen des Hahnes II würde ein Teil der Thoulet'schen Lösung in den Cylinder $C_{1}$ gelassen und von neuem evacuiert, bis keine Blasen mehr in der Flüssigkeit aufstiegen. Die Correclion der Concentration der Lüsung geschah in Cylin$\operatorname{der} C_{2}$. Die Bestimmung der specifischen Gewichte wurde auch zuweilen in großen, dickwandigen Reagensgläsern mit Erfolg ausgeführt.

Was nun die für die specifischen Gewichte gefundenen Werte betrifft, so muß ich bemerken, daß sie sich nicht durch große Genauigkeit auszeichnen. Es ist sehr schwer, specifische Gewichte von so fein-pulverigeu 
und außerdem noch so leicht zersetzlichen Substanzen einigermaßen genau zu bestimmen. Die in die Tabellen eingelragenen Werte sind Mittelwerte aus je drei Beobachtungen. Die Thoulet'sche Lösung wurde so lange corrigiert, bis nichts mehr von der Substanz auf dem Boden saß, d. h. es wurde immer das specifische Gewicht der schwersten Teilchen bestimmt. Die Bestimmungen wurden mit der Mohr'schen Wage ausgeführt. Ich lasse die einzelnen Bestimmungen hier folgen:

Tabelle I.

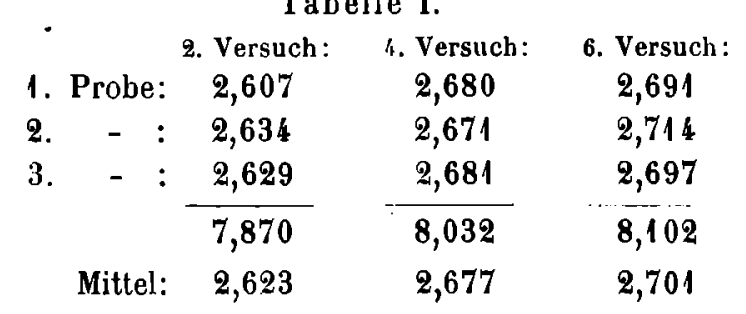

Tabelle IIa.

7. Versuch: 9. Versuch: 11. Versuch:

1. Probe: 2,615 2,688 2,722

2. $-: \begin{array}{rrr}2,605 & 2,692 & 2,735\end{array}$

3. $-\frac{2,606}{7,826} \quad \frac{2,702}{8,082} \quad-\frac{2,743}{8,200}$

Mittel: $\quad 2,609 \quad 2,694 \quad 2,733$

Tabelle IIb.

12. Versuch: 14. Versuch: 16. Versuch:

1. Probe: 2,624 2,693 2,729

2. $\quad-\quad: \quad 2,599 \quad 2,678 \quad 2,756$

3. - : $\frac{2,599}{7,822} \quad \frac{2,683}{8,054} \quad \frac{2,753}{8,238}$

Mittel: $\quad 2,607 \quad 2,685 \quad 2,746$

Tabelle III a.

17. Versuch: 19. Versuch: 21. Versuch:

1. Probe: $2,618 \quad 2,679 \quad 2,778$

2. $\quad$ - : $2,630 \quad 2,671 \quad 2,805$

3. - : $\frac{2,622}{7,870} \quad \frac{2,714}{8,064} \quad \frac{2,807}{8,390}$

Mittel: $\quad 2,623 \quad 2,688 \quad 2,797$ 
Über isom. Mischsalze von wasserfreiem Kálk-, Magnesium- u. Eisencarbonat. 271

Tabelle IIIb.

Q2. Versuch: 24. Versuch: 26. Versuch:

\begin{tabular}{rrrrr} 
1. Probe: & 2,618 & 2,721 & 2,831 \\
$2 . \quad-:$ & 2,630 & 2,692 & 2,801 \\
$3 . \quad-:$ & 2,622 & 2,701 & 2,807 \\
\cline { 3 - 4 } & $\mathbf{7 , 8 7 0}$ & $\mathbf{8 , 1 1 4}$ & $\mathbf{8 , 4 3 9}$ \\
& Mittel: & 2,623 & 2,705 & $\mathbf{2 , 8 1 3}$
\end{tabular}

Die auf angegebene Weise gefundenen Mittelwerte sind in die letzte Colonne der Tabellen eingetragen.

Die so gefundenen specifischen Gewichte werde ich nun benutzen, um die Frage zu entscheiden, in welcher Modification der kohlensaure Kalk in den Mischcarbonaten auftritt. Zu diesem Zwecke ist es nölig, die specifischen Gewichte der wasserfreien Substanzen zu berechnen.

Ist $d$ das specifische Gewicht der wasserhalligen Substanz, $d_{1}$ dasjenige der wasserfreien und $a$ der Procentgehalt an Wasser, so kann man $d_{1}$ berechnen nach der Formel

$$
\begin{aligned}
100 & =\frac{100-a}{d_{1}}+a \\
\text { oder } \quad d_{1} & =\frac{100-a}{\frac{100}{d}-a} . a
\end{aligned}
$$

Tabelle IV.

\begin{tabular}{|c|c|c|c|c|c|c|c|c|c|}
\hline \multirow[t]{2}{*}{$\mathrm{Nr}}$. & \multicolumn{3}{|c|}{$\begin{array}{c}\text { Analyse, berechnet } \\
\text { auf } 100\end{array}$} & \multicolumn{2}{|c|}{$\begin{array}{c}\text { Analyse, } \\
\text { berechnet auf } \\
\text { wasserfreie S. }\end{array}$} & \multicolumn{2}{|c|}{$\begin{array}{l}\text { Mol.-Propor- } \\
\text { tionen, } \\
\text { ber. auf } 10\end{array}$} & \multicolumn{2}{|c|}{ Spec. Gewichte } \\
\hline & $\mathrm{CaCO}_{3}$ & $\mathrm{FeCO}$ & $\mathrm{H}_{2} \mathrm{O}$ & $\mathrm{CaCO}_{3}$ & $\mathrm{FeCO}$ & $\mathrm{CaCO}_{3} \mid$ & $\mathrm{FeCO}$ & $\underset{\text { beobachtet }}{\mathrm{H}_{2} \mathrm{O}}$ & $\begin{array}{l}\text { ohne } \mathrm{H}_{2} \mathrm{O} \\
\text { berechnet }\end{array}$ \\
\hline 6 & 51,04 & 44,72 & 4,24 & 53,30 & 46,70 & $\begin{array}{l}5,697 \\
5,7\end{array}$ & $\begin{array}{l}4,303 \\
4,3\end{array}$ & 2,701 & 2,921 \\
\hline 7 & 78,71 & 18,50 & 2,73 & 80,92 & 19,08 & $\begin{array}{l}8,911 \\
8,3\end{array}$ & $\begin{array}{l}1,689 \\
1,7\end{array}$ & 2,609 & 2,732 \\
\hline 9 & 52,88 & 42,55 & 4,57 & 35,41 & 44,59 & $\begin{array}{l}5,904 \\
5,9\end{array}$ & $\begin{array}{l}4,096 \\
4,1\end{array}$ & 2,694 & 2,932 \\
\hline 11 & 32,49 & 61,70 & 5,81 & 34,49 & 65,51 & $\begin{array}{l}3,792 \\
3,8\end{array}$ & $\begin{array}{l}6,208 \\
6,2\end{array}$ & 2,733 & 3,060 \\
\hline 12 & 85,25 & 12,24 & 2,51 & 87,44 & 12,56 & $\begin{array}{l}8,899 \\
8,9\end{array}$ & $\begin{array}{l}1,101 \\
1,1\end{array}$ & 2,607 & 2,713 \\
\hline 14 & 58,93 & 37,02 & 4,05 & 64,43 & 38,57 & $\begin{array}{l}6,488 \\
6,5\end{array}$ & $\begin{array}{l}3,512 \\
3,5\end{array}$ & $2,68: 5$ & 2,891 \\
\hline 16 & 32,49 & 61,70 & 5,81 & 34,49 & 65,31 & \begin{tabular}{l|l}
3,792 \\
3,8
\end{tabular} & $\begin{array}{l}6,208 \\
6,2\end{array}$ & 2,733 & 3,060 \\
\hline
\end{tabular}

Zusammenstellung der zur graphischen Darstellung (Fig. 7) verwandten Substanzen. 
Der Einfachheit halber beschränke ich mich auf die magnesiafreien Substanzen, welche ich in der Tabelle IV auf S. 271 zusammengestellt habe. In die ersten drei Colonnen sind die Analysenwerte, auf 100 berechnet, eingetragen worden. Die nāchsten beiden Spalten enthalten die procentische Zusammensetzung der wasserfreien Substanzen, und hierauf folgen die auf 10 berechneten Molekularproportionen. In der vorletzten Colonne sind die beobachteten specifischen Gewichte der wasserhaltigen $(d)$ und in der letzten die berechneten der wasserfreien Substanzen $\left(d_{1}\right)$ angefübrt.

Das specifische Gewicht des Calciumcarbonats ermittele ich nun aus den für $d_{1}$ gefundenen Werten durch Extrapolation. $\mathrm{Zu}$ diesem $\mathrm{Zwecke}$

Fig. 7 .

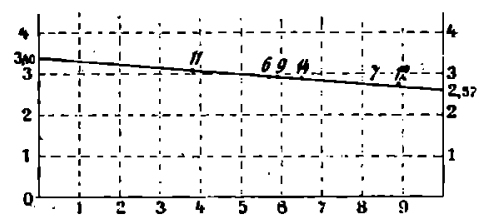
trage ich die Molekularproportionen und specifischen Gewichte in ein Coordinatensystem (Fig. 7) ein, und zwar auf die Abscisse das Verhăltnis von Calciumcarbonat zu Eisencarbonat, bezogen auf 10, und auf die Ordinate die specifischen Gewichte $\left(d_{1}\right)$. Es zeigt sich nun, daß die gefundenen Punkte fast genau in einer Geraden liegen. Bringe ich diese Gerade mit den im Anfangs- und Endpunkt der $\Lambda$ bscisse errichtelen Ordinaten zum Schnitt, so geben mir diese Schnitlpunkte das specifische Gewicht des reinen Calciumcarbonats einerseils und das des reinen Eisencarbonats andererseits an. Aus der Fig. 7 ergeben sich die Werte:

$$
\begin{array}{cll}
\text { für Eisencarbonat: } & \mathbf{3 , 4 0}, \\
\text { \ Calciumcarbonat: } & \mathbf{2 , 5 7} .
\end{array}
$$

Der für das Calciumcarbonat gefundene Wert kommt dem specifischen Gewicht des Vaterits, welches nach Bütschli $\mathbf{2 , 5 4}$ beträgt, sehr nahe. Es muß also angenommen werden, daß das Calciumcarbonat in den Mischsalzen als Vaterit vertreten ist.

\section{Das Verhalten von Aragonit, Calcit and Vaterit, sowie von Calcinm - Kagnesiumcarbonaten gegen Eisenoxydulsalzlösungen.}

Über das Verhalten von Aragonit und Calcit gegen eine große Anzahl von Metallsalzen sind eingehende Untersuchungen angestellt worden von Meigen ${ }^{4}$ ), E. Werner ${ }^{5}$ ) und A. Lange ${ }^{6}$ ). Das Resultat dieser Untersuchungen war, daß sich diese beiden Modificationen des bohlensauren Kalkes bei allen Reactionen verschieden verhalten, wenn auch die Unterschiede zum Teil nur gering sind. Diese Untersuchungen wurden später fortgesetzt von L. Gaßner ${ }^{7}$ ), dessen Arbeit ich einige Tatsachen über das Verhalten von Calcit und Aragonit gegen Eisenoxydulsalzlüsungen entnehme. Als 
Eisenoxydulsalz verwendete der Verfasser ebenfalls das Mohr'sche Salz. Er löste $39 \mathrm{~g}$ in $1000 \mathrm{ccm}$ Wasser und versetzte $350 \mathrm{ccm}$ dieser $\frac{1}{10}$-molekularen Lösung mit $3 \mathrm{~g}$ Aragonit und weitere $350 \mathrm{ccm}$ mit $3 \mathrm{~g}$ Calcit. Die von Aragonit gefïllte Menge Eisen betrug nach 15 Stunden 49,0\%, nach 4 Tagen 76,00! nach 11 Tegen $93,9 \%$ und nach 17 Tagen 96,6\%. Für den Calcit betrug die gefällte Menge Eisen nach 15 Stunden 22,3\%, nach 4 Tagen 24,4\%, nach 11 Tagen $36,2 \%$ und nach 17 Tagen 49,1\%.

Das Resultat ist folgendes: Eisenoxydulsalzlösungen werden von Aragonit bedeutend stärker gefällt als von Calcit. Während der von Aragonit gebildete Niederschlag nach Beginn der Reaction dunkelgrün ist, zeigt der von Calcit erzeugte Niederschlag eine gelbe Farbe.

Ich habe nun ganz analoge Versuche mit Calcit, Aragonit und Vaterit ausgeführt. Den hierzu verwandten Vaterit stellte ich auf Veranlassung des Herrn Prof. Linck gemeinsam mit Herrn Spangenberg in Anlehnung an die Angaben von Bütschli ${ }^{8}$ ) her. $300 \mathrm{~g}$ Kaliumcarbonat wurden in $600 \mathrm{ccm}$ Wasser gelöst. Diese Lösung wurde mit $200 \mathrm{ccm}$ einer doppeltnormalen Chlorcalciumlösung versetzt. Nachdem der Niederschlag krystallinisch geworden war, wurde er rasch abfiltriert, zuerst mit verdünnter Kaliumcarbonatlösung bis zum Verschwinden der Chlorreaction, dann mit Wasser und schließlich mit absolutem Alkohol ausgewaschen und im Trockenschrank bei ca. $80^{\circ}$ getrocknet, wobei keine Umwandlung eintrat. Der Niederschlag bestand aus außerordentlich kleinen, sehr schwach doppelbrechenden Sphärolithen. Das specifische Gewicht wurde nicht genau bestimmt, jedoch wurde festgestellt, daß es unter 2,55 lag.

Eine Probe von dem Niederschlag wurde 2 Tage lang unter destilliertem Wasser in einem Thermostaten auf einer Temperatur von ca. $50^{0}$ erhalten, wobei der größte Teil der Sphärolithe in Calcitrhomboëder verwandelt wurde. Mit Kobaltsolution gekocht, trat bereits nach wenigen Secunden Violettfärbung ein.

$\mathrm{Zu}$ den folgenden Versuchen wurde ebenfalls eine $\frac{1}{10}$ molekulare Lösung von Mohr'schem Salz verwandt, enthaltend 39,224 g Salz in 1 Liter. Um während der Versuche die Oxydation müglichst zu vermeiden, wurde Wasser benutzt, welches durch Auskochen von Luft befreit worden war.

Die Versuche wurden ausgeführt in kleinen Erlenmeyer-Kolben, welche, bis fast zum Rande gefüllt, $110 \mathrm{ccm}$ faßten, sodaß sich beim Verschließen der Kölbchen über der Flüssigkeit nur eine geringe Luftmenge befand. Vier solcher Külbchen wurden nun beschickt mit $1 \mathrm{~g}$ natürlichen, fein pulverisierten Calcit, $1 \mathrm{~g}$ gefällten Calcit, $1 \mathrm{~g}$ natürlichen, ebenfalls fein pulverisierten Aragonit und $1 \mathrm{~g}$ nach der angegebenen Methode hergestellten Vaterit. Hierauf wurden zu jeder Probe $110 \mathrm{ccm}$ der oben beschriebenen Mohr'schen Salzlösung gebracht und sämtliche vier Kölbchen mit Gummistopfen luftdicht verschlossen. Sofort nach dem Zusammenbringen der 
Substanzen .nahm die Flüssigkeit beim Calcit eine gelbe, beim Aragonit und Vaterit eine dunkelgrüne Fărbung an, und zwar schien die Färbung beim Aragonit intensiver zu sein als beim Vaterit. Nach ca. einer halben Stunde hatten sich am Boden der Gefäße Niederschlüge von gleicher Farbe abgesetzt, während die Flüssigkeit wieder vollkommen klar geworden war. Alle drei Stunden wurden sämtliche vier Külbchen tüchtig geschültelt und nach Verlauf von 46 Stunden die Niederschläge abfiltriert. Die Abnahme des Eisengehalts in der Lösung wurde durch Titration mit Kaliumpermanganat ermittelt. Die Titrationen ergaben folge Resultate:

$20 \mathrm{ccm}$ der Ausgangslüsung

20 - des Filtrats vom natürl. Calcit

20 - - - - gefällten -

$20-\quad-\quad-$ Aragonit

20 - - - - Vaterit

Hieraus ergibt sich:

In 46 Stunden fällt der natürliche Calcit $12,61 \% \mathrm{Fe}$, gefällte Calcit Aragonit Vaterit

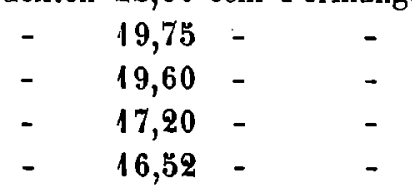
$13,27 \% \mathrm{Fe}$, $23,89 \% \mathrm{Fe}$, $26,90 \% \mathrm{Fe}$. brauchten 22,60 ccm Permanganat,

Der Vaterit fällt also von den drei Modificationen des kohlensauren Kalkes das Eisen aus Oxydulsalzlösungen am schnellsten.

Ich möchte nun zum Schluß noch einige von mir angestellte Versuche über"die Einwirkung von Eisenoxydulsalzlösungen auf Calcium-Magnesiumcarbonate beschreiben.

Ich füllte ein kurzes Reagensgläschen mit einer Mohr'schen Salzlösung von bestimmter Concentration bis ziemlich zum Rande. Die Lüsung wurde für jeden einzelnen Versuch besonders hergestellt, und zwar wurde Wasser benutzt, welches vorher durch Auskochen vollständig von Luft befreit worden war. In diese Lüsung hinein gab ich ungefähr $0,5 \mathrm{~g}$ eines Calcium-Magnesiumcarbonats, das durch Zusammenbringen von $50 \mathrm{ccm}$ einer doppeltnormalen Magnesiumchloridlüsung, $100 \mathrm{ccm}$ einer einhalbnormalen Calciumchloridlösung und $150 \mathrm{ccm}$ einer $1 \frac{1}{2}$ normalen Ammoniumsesquicarbonatlösung bei einer Temperatur von 200 hergestellt worden war. Hierauf verschloB ich das Gläschen mit einem Korkstopfen, und um das Eindringen von Luft zu verhindern, bedeckte ich den Kork mit einer Paraffinschicht. Das Gläschen wurde nun so gelegt, daß immer eine möglichst große Oberfläche der Substanz mit der Lösung in Berührung. war. Außerdem wurde täglich 3-4 mal tüchtig geschüttelt. Bei den drei folgenden Versuchen wurde das Calcium-Magnesiumcarbonat von der Zusammensetzung : 
Über isom. Mischsalze von wasserfreiem Kalk-, Magnesium- u. Eisencarbonat. 275

$$
\begin{array}{cl}
84,55 \% & \mathrm{CaCO}_{3} \\
12,39 \% & \mathrm{MgCO}_{3} \\
2,71 \% & \mathrm{H}_{2} \mathrm{O} \\
99,65 \% &
\end{array}
$$

benutzt.

Versuch 1. Die Concentration der Mohr'schen Salzlösung war doppeltnormal. Die Temperatur wurde auf $20^{\circ}$ erhalten. Die Dauer des Versuchs betrug \& Tage. Die Substanz wurde rasch abfiltriert, mit Wasser und absolutem Alkohol ausgewaschen und nach dem oben angegebenen Verfahren getrocknet. Die Analyse ergab:

$$
\begin{array}{cc}
82,11 \% & \mathrm{CaCO}_{3} \\
12,24 \% & \mathrm{MgCO}_{3} \\
3,14 \% & \mathrm{FeCO} \\
2,65 \% & \mathrm{H}_{2} \mathrm{O} \\
100,14 \% &
\end{array}
$$

Versuch 2. Die Concentration der Mohr'schen Salzlösung war normal. Die Dauer des Versuchs betrug 11 Tage, die Temperatur ebenfalls 200. Das Ergebnis der Analyse war:

$$
\begin{array}{ccc}
79,75 \% & \mathrm{CaCO}_{3} \\
11,79 \% & \mathrm{MgCO}_{3} \\
5,51 \% & \mathrm{FeCO} 3 \\
\mathbf{2 , 6 9 \%} & \mathrm{H}_{2} \mathrm{O} \\
\hline \mathbf{9 9 , 7 4 \%} &
\end{array}
$$

Versuch 3. Die Mohr'sche Salzlösung war wieder doppeltnormal. Der Versuch dauerte 11 Tage bei einer Temperatur von 200. Analyse:

$$
\begin{array}{cl}
78,04 \% & \mathrm{CaCO}_{3} \\
10,80 \% & \mathrm{MgCO}_{3} \\
8,46 \% & \mathrm{FeCO}_{3} \\
\mathbf{2 , 5 9 \%} \% & \mathrm{H}_{2} \mathrm{O} \\
99,89 \% &
\end{array}
$$

Um zu zeigen, in welchem Maße Calcium- und Magnesiumcarbonat aus dem Mischsalz durch Eisencarbonat verdrängt werden, habe ich die Abnahme des Calciumcarbonals bezw. Magnesiumcarbonats in Procenten berechnet und die Resultate in die Tabelle V auf S. 276 eingetragen. Es gibt also z. B. die Zahl 2,886 in der 7. Spalte an, um wieviel Procente, bezogen auf die Zahl $\mathbf{8 4 , 5 5}$ in der dritten Spalte, der Gehalt an Calciumcarbonat abgenommen hat. In die erste Zeile der Tabelle ist die Zusammensetzung des den Versuchen zugrunde gelegten Calcium-Magnesiumcarbonats eingetragen worden.

Die Versuche 1 und 3 bilden eine Reihe, bei welcher die Concentration 
Tabelle V.

Versuche über die Einwirkung von Calcium-Magnesiumcarbonaten auf Eisenoxydulsalzlösungen.

\begin{tabular}{|c|c|c|c|c|c|c|c|c|}
\hline \multirow{2}{*}{ Nr. } & \multirow{2}{*}{$\begin{array}{c}\text { Dauer } \\
\text { des Versuchs }\end{array}$} & \multirow{2}{*}{$\begin{array}{l}\text { Concentr. der } \\
\text { Mohr'schen } \\
\text { Salzlösung }\end{array}$} & \multicolumn{4}{|c|}{ Analyse in $\%$} & \multicolumn{2}{|c|}{ Abnahme in $\%$} \\
\hline & & & $\mathrm{CaCO}_{3}$ & $\mathrm{MgCO}_{3}$ & $\mathrm{FeCO}$ & $\mathrm{H}_{2} \mathrm{O}$ & $\mathrm{CaCO}$ & $\mathrm{MgCO}_{3}$ \\
\hline & - & - & 84,55 & 12,39 & - & 2,71 & - & - \\
\hline 1 & 4 Tage & $2 n$ & 82,11 & 12,24 & 3,14 & 2,65 & 2,886 & 1,211 \\
\hline 2 & 11 Tage & $n$ & 79,75 & 11,79 & 5,51 & 2,69 & 5,677 & 4,843 \\
\hline 3 & 11 Tage & $2 n$ & 78,04 & 10,80 & 8,46 & 2,59 & 7,70 & 12,83 \\
\hline
\end{tabular}

der Mohr'schen Salzlösung constant ist, die Versuchsdauer dagegen sich ändert. Die Abnahme des Calciumcarbonats beträgt 2,886 und 7,70\%, diejenige des Magnesiumcarbonats 1,211 und 12,83\%. Es geht also hieraus hervor, daß die Abnahme des Magnesiumcarbonats in viel stärkerem Maße steigt, als die des Calciumcarbonats. Es wird also das Magnesium rascher aus dem Mischcarbonat verdrängt, als das Calcium.

In welcher Modification der kohlensaure Kalk in dem diesen Versuchen zugrunde gelegten Calcium-Magnesiumcarbonat vertreten ist, werden die von Schmidt angestellten Untersuchungen zeigen, welche zur Zeit noch nicht abgeschlossen sind.

\section{Zusammenfassung.}

Fassen wir die wichtigsten Resultate dieser Arbeit noch einmal zusammen, so ergibt sich folgendes:

Das Eisencarbonat zeigt größere Neigung mit Calciumcarbonat isomorphe Mischsalze zu bilden als das Magnesiumcarbonat. Calciumcarbonat und Eisencarbonat mischen sich in allen Verhältnissen zu vollkommen einheitlichen Substanzen. Das Magnesiumcarbonat dagegen tritt nur bis zu einem geringen Betrage in das Mischsalz ein. Bei der Fällung von Kalk-, Magnesia- und Eisenoxydulsalzen mit Ammoniumsesquicarbonat wird das Eisen vollständig ausgefällt, während etwas Kalk und viel Magnesia in Lösung bleibt. Das Calciumcarbonat tritt in den Mischcarbonaten als Vaterit auf.

Unter dem Mikroskope betrachtet, stellen sämtliche Mischsalze vollkommen einheitliche Substanzen dar. Sie bestehen aus Sphärolithen von verschiedener Größe. Der Eisengehalt ist auf die Größe der Sphärolithe von großem Einfluß. Je höher der Eisengehalt, desto kleiner sind die Sphärolithe. Vom Eisengehalt hăngt auch die äußere Form ab. Eisenreichere Sphärolithe haben vollkommene Kugelgestalt und sind scharf begrenzt, eisenarme dagegen weichen von der Kugelgestalt oft beträchtlich 
Über isom. Mischsalze von wasserfreiem Kalk-, Magnesium- u. Eisencarbonat. 277

$a b$, und ihre Begrenzung ist meist unscharf und zackig. Concentrischschalige und radialfaserige Structuren sind am deutlichsten an eisenarmen Substanzen zu bemerken. Bei wachsendem Eisengehalt wird besonders die radialfaserige Structur immer feiner und schwerer sichtbar.

Die Sphärolithe schließen sehr oft einen trüben Kern ein, der zuweilen Krystallhabitus zeigt. Solche Substanzen geben häufig in Salzsäure gelöst mit Chlorbaryum eine Trübung, woraus folgt, daß die Kerne wahrscheinlich zum Teil aus Gypskryställchen bestehen. Gerade solche Sphärolithe, welche einen Kern enthalten, zeigen die beiden Struclurarten am deutlichsten. Sie sind dagegen vollkommen unsichtbar bei kalkfreien Sphärolithen.

Sämtliche Sphärolithe sind doppeltbrechend und zwar negativ. Zwischen gekreuzten Nicols zeigen sie farbige Ringe und ein schwarzes Kreuz.

Von den drei Modificationen des kohlensauren Kalkes füllt die Valer'sche das Eisen aus Oxydullösungen am schnellsten. Läßt man Calcium-Magnesiumcarbonate auf Eisenoxydulsalzlösungen einwirken, so fällt das Magnesiumcarbonat das Eisen schneller als das Calciumcarbonat.

Vorliegende $\Lambda$ rbeit wurde im Mineralogischen Institute der Universilät Jena ausgeführt. Es ist mir eine angenehme Pflicht, meinem hochverehrten Lehrer, Herrn Geh. Iofrat Prof. Dr. G. Linck; welcher mir die Anregung zu meiner Arbeit gab, für das meinem Studium und speciell meinen Untersuchungen stets entgegengebrachte fürdernde Interesse meinen herzlichsten Dank auszusprechen. Zu großem Danke verpflichtet bin ich auch Herrn Privatdocenten Dr. Marc für zahlreiche mir wertvolle Ratschläge, sowie Herrn Dr. Kühler, wissenschaftlicher Mitarbeiter bei der Firma »Carl $Z$ ei $B$ *, für die freundliche Herstellung der Mikrophotographien.

Jena, Mineralogisches Institut, im December 1910.

\section{Literaturverzeichnis.}

1. Linck, Über die Entstehung der Dolomile. Monatsber. d. deutsch. geol. Ges. 1909 , Nr. 5, 230.

2. Dammer, Handbuch der anorg. Chemic 3, 363.

3. Schmidt, Über isomorphe Mischcarbonate von Kalk und Magnesia. Manuscript.

4. W. Meigen, Beiträge zur Kenntnis des kohlensauren Kalkes. Ber. d. Naturf.Ges. zu Freiburg i. Br. 1902, 13, 10-94. Ausz. diese Zeitschr. 40, 524. Beitr. zur Kenntnis des kohlensauren kalkes. II. und Ill. Ber. d. Naturf.Ges. zu Freiburg 1. Br. 1905, 15, 38-74. Ausz. diese Zeitschr. 44, 312.

5. E. Werner, Beiträge zur Kenatnis des kohlensauren Kalkes. Inaugur.-Dissert. Freiburg i. Br. 1903.

6. A. Lange, Über das Verhalten von kohlensaurem Kalk zu Kobaltsalzen. Inaug.Diss. Freiburg i. Br. 1904.

7. L. Gaßner, Weitere Beiträge zur Kenntnis des kohlensauren Kalkes. Inaug.Diss. Freiburg i. Br. 1906.

8. 0 . Bütschli, Untersuchungen über organische Kalkgebilde. Abhandl. der kgl. Ges. d. Wissensch. zu Göttingen. Math.-physik. Klasse, neue lolge 6, Nr. 3. 